\title{
In Pursuit of New Imprinting Syndromes by Epimutation Screening in Idiopathic Neurodevelopmental Disorder Patients
}

\author{
Sonia Mayo, Sandra Monfort, Mónica Roselló, Silvestre Oltra, \\ Carmen Orellana, and Francisco Martínez \\ Unidad de Genética y Diagnóstico Prenatal, Hospital Universitario y Politécnico La Fe, Avenida de Campanar 21, \\ 46009 Valencia, Spain \\ Correspondence should be addressed to Francisco Martínez; francisco@gva.es
}

Received 21 October 2014; Revised 4 May 2015; Accepted 11 May 2015

Academic Editor: Marco Fichera

Copyright (c) 2015 Sonia Mayo et al. This is an open access article distributed under the Creative Commons Attribution License, which permits unrestricted use, distribution, and reproduction in any medium, provided the original work is properly cited.

Alterations of epigenetic mechanisms, and more specifically imprinting modifications, could be responsible of neurodevelopmental disorders such as intellectual disability (ID) or autism together with other associated clinical features in many cases. Currently only eight imprinting syndromes are defined in spite of the fact that more than 200 genes are known or predicted to be imprinted. Recent publications point out that some epimutations which cause imprinting disorders may affect simultaneously different imprinted loci, suggesting that DNA-methylation may have been altered more globally. Therefore, we hypothesised that the detection of altered methylation patterns in known imprinting loci will indirectly allow identifying new syndromes due to epimutations among patients with unexplained ID. In a screening for imprinting alterations in 412 patients with syndromic ID/autism we found five patients with altered methylation in the four genes studied: MEG3, H19, KCNQ1OT1, and SNRPN. Remarkably, the cases with partial loss of methylation in KCNQ1OT1 and SNRPN present clinical features different to those associated with the corresponding imprinting syndromes, suggesting a multilocus methylation defect in accordance with our initial hypothesis. Consequently, our results are a proof of concept that the identification of epimutations in known loci in patients with clinical features different from those associated with known syndromes will eventually lead to the definition of new imprinting disorders.

\section{Introduction}

Intellectual disability (ID) is a complex disease which affects $2 \%$ of our population. Known genetic and environmental causes are responsible for a large proportion of the cases; however the etiology in many patients remains unknown because of the elevated clinical and genetic heterogeneity. Deregulation of epigenetic mechanisms in brain development and neuronal plasticity may be associated with a wide spectrum of neurological and psychiatric disorders [1-4]. In fact, a growing number of syndromic forms of ID are caused by mutations in genes involved in epigenetic regulation as Sotos or Rett syndrome among others. However, these mutations only account for a small number of cases. There are evidences that aberrant epigenetic mechanisms play a role in autism and other neurodevelopmental disorders [5-7]. Also, genomescale approaches to study the epigenetic alterations point out a possible association of global hypomethylation and different neurological disorders as schizophrenia or bipolar disorder $[8,9]$. However, high-throughput methodology is expensive, time-consuming, and of complex and controversial interpretation in many occasions [10, 11].

Genomic imprinting is an epigenetic mechanism by which gene expression is regulated in a parent-of-originspecific manner [12]. There are 95 proven and 114 predicted imprinted genes in the human genome (Geneimprint database). Furthermore, many of these genes are expressed in the central nervous system, among other tissues, and are predicted to act as transcriptional regulators in development. Nevertheless, the clinical consequences of the loss of function of these genes, due to mutation or epimutation, are largely unknown. Currently, there are 8 recognised imprinting syndromes associated with growth and behavioural disorders: Silver-Russell syndrome (SRS), Beckwith-Wiedemann syndrome (BWS), Prader-Willi syndrome (PWS), Angelman syndrome (AS), transient neonatal diabetes (TNDM), 
maternal uniparental disomy 14-like (UPD(14)mat) and UPD(14)pat-like syndromes, and pseudohypoparathyroidism 1B (PHP1B). The mechanisms that result in altered imprinted gene expression are diverse. Four different mechanisms have been described: large deletions or duplications of regions containing imprinted genes, DNA mutations in an imprinted gene, uniparental disomy, and epimutations. Each different cause is associated with varying recurrent risks; for example, epimutations or de novo deletions usually imply very low risk of recurrence for parents and other relatives, whereas some deletions and point mutations can have a $50 \%$ recurrence.

Recent publications claim that several genetic variants manifest a parent-of-origin effect in autism [6, 13, 14]. Moreover, it is increasingly evident that epimutations leading to imprinting disorders in some instances may affect not one but several imprinted loci throughout the genome, suggesting that imprinting-specific DNA-methylation may have been altered more globally due to unknown factors [1521]. Phenotypic differences of these cases with the classical imprinting syndromes may be present or not and can be attributed to abnormal DNA-methylation elsewhere.

Based on these findings, we hypothesize that many of the imprinted genes of unknown clinical consequences may be responsible for neurodevelopmental disorders when epimutated, associated or not with other congenital anomalies. The detection of altered methylation patterns in known imprinted loci will allow the identification of new syndromes due to multilocus epimutations among patients with unexplained neurodevelopmental disorders. To asses this hypothesis, we searched for aberrant methylation at four imprinted loci (SNRPN, H19, KCNQ1OT1, and MEG3) in a series of 412 patients with intellectual disability using a methylation analysis affordable for any laboratory. We found five cases with alteration of methylation: two alterations in the methylation pattern of MEG3 as a consequence of paternal or maternal uniparental disomy for chromosome 14, one hypermethylation of H19 (due to paternal 11p duplication), one partial loss of methylation in KCNQ1OT1, and one partial loss of methylation in SNRPN.

\section{Patients and Methods}

2.1. Patient Samples. DNA samples of 412 patients were analyzed in this study. They were recruited for genetic investigation of unexplained ID and/or autism during more than 10 years (October 2001-July 2013). This research was carried out according to the principles of the Declaration of Helsinki. Informed consent, approved by the Hospital Ethics Committee, was obtained from all the parents of the children who participated in the study.

Genomic DNA was isolated from peripheral blood. Former samples (up to 2009) were extracted by the phenol extraction protocol described by [22]. Since 2010 the DNA extraction was performed using QIAamp DNA Mini Kit and the QIAcube automated extractor (QIAGEN, Hilden, Germany). DNA quality and concentration were measured using the NanoDrop ND-1000 Spectrophotometer (NanoDrop Technologies, Rockland, DE, USA) and were stored at $-20^{\circ} \mathrm{C}$.
The selection criteria of the patients, in addition to the intellectual disability or autism spectrum disorders (ASD), were the presence of congenital abnormalities, dysmorphic features, and/or a positive family history for neurodevelopmental disorders or congenital abnormalities. None of the patients had a specific genetic diagnosis when recruited. Genomic rearrangements' analyses by array CGH were performed in all these patients as part of our investigation. The methylation study was systematically carried out, as a blind test, that is, not taking into account previous genetic results. Once the analysis was performed, all the pieces of information were gathered together for the phenotypegenotype correlation.

2.2. Previous Tests. Genomic rearrangements were studied by oligonucleotide-based genome-wide array CGH (44K, G4426B; Agilent Technologies, Palo Alto, CA, USA), a targeted custom array for ID and autism (manuscript in preparation; Agilent Technologies), SNP-array (Affymetrix Genome-Wide Human SNP 6.0 Array, Santa Clara, CA, USA), MLPA (MRC-Holland), and/or FISH (telomeric commercial probes TelVysion, Vysis, Downers Grove, IL, USA), using the recommended protocols by the manufacturer with minor modifications [23-25].

The data related to array results discussed in this paper have been deposited in NCBI's Gene Expression Omnibus [26] and are accessible through GEO series accession number GSE62440 (http://www.ncbi.nlm.nih.gov/geo/query/acc .cgi? acc $=$ GSE62440).

2.3. Methylation Test. Based on a multiplex amplification and quantification methylation test previously described in Martínez et al. [27], we performed a screening for DNAmethylation alterations in four differentially methylated regions (DMRs) associated with specific imprinting syndromes: KCNQ1OT1 (11p15; BWS), H19 (11p15; SRS and BWS), SNRPN (15q12; PWS and AS), and MEG3 (14q32; UPD14pat and UPD14mat), in our series of patients.

$50 \mathrm{ng}$ of genomic DNA was digested with 10 units of the methylation sensitive enzyme HpaII (Fermentas), while another aliquot was used as undigested control. Both were incubated at $37^{\circ} \mathrm{C}$ for one hour, followed by heat inactivation at $94^{\circ} \mathrm{C}$ for 3 minutes. Undigested and digested DNAs were used as a template for a FAM-labelled multiplex PCR under semiquantitative conditions (see Supplementary Table 1 in Supplementary Material available online at http://dx.doi.org/10.1155/2015/341986). Resulting PCR products were analysed on an ABI-3130XL genetic analyser (Applied Biosystems) and each peak area was divided by the sum of all peak areas of that sample (relative area) and then normalized to the corresponding averaged relative areas obtained on control samples. Data analysis was performed in an Excel spreadsheet (Microsoft Office 2007).

2.4. Confirmation Tests. DNA samples with a relative value of methylation in the screening outside the $0.8-1.2$ normal range were confirmed with alternative techniques. KCNQ1OT1 
and $H 19$ alterations were validated by methylation specific multiplex ligation-dependent probe amplification (MSMLPA) using SALSA ME030; and SNRPN alterations were validated with SALSA ME028 (MRC-Holland, Amsterdam, Netherlands). The technical protocols and the analysis were performed as recommended by the manufacturer (MRCHolland).

The possibility of a uniparental disomy was tested by segregation analysis of different microsatellite markers from the corresponding loci in the patients and their parents DNA samples (reagents and PCR conditions at Supplementary Table 2).

All genomic coordinates given below are based on Human Feb. 2009 assembly (GRCh37/hg19).

\section{Results}

In the screening for imprinting alterations in the 412 patients with neurodevelopmental disorders we have found five cases with different alterations of methylation.

3.1. Patient 1. The patient presents an $80 \%$ loss of methylation at KCNQ1OT1 with a normal gene dosage (Figure 1). The results were confirmed by MS-MLPA (SALSA ME030). Segregation analysis of chromosome 11 markers discarded the UPD as the genetic mechanism responsible of the altered methylation pattern. Screening for dosage alterations was performed by oligo-CGH-array with no relevant findings (GSM1527006).

This case was previously published with the clinical description of the patient [28]. In addition to motor and language delay and mild intellectual disability, he presents some clinical features resembling Sotos syndrome such as overgrowth, frontal bossing, sparse hair in the frontoparietal area, macrocephaly, and dolichocephaly.

3.2. Patient 2. This female patient shows hypermethylation of H19 and an increased dosage of H19 and KCNQ1OT1 in chromosome 11 (Figure 1). Previous genetic analyses detected a complex rearrangement: a $3.1 \mathrm{Mb} 11$ pter-p15.4 duplication and a $3.7 \mathrm{Mb} 4$ pter-p16.2 deletion due to an unbalanced translocation inherited from her father (arr [hg19] 4 p16.3 $(1-3,770,271) \times 1$ pat, 11p15.5p15.4(1-3,381,999) × 3 pat $)$ (GSM1527007). Both results are in agreement (Table 1).

She was the first-born child of unrelated healthy parents, a 27-year-old mother and a 28-year-old father. She was born at term by normal delivery. Her birth weight was 3,360 $\mathrm{g}$ (7550th percentile) and her length was $52 \mathrm{~cm}$ (90th percentile). Prenatal cytogenetic analysis was performed with normal results. On physical examination at 8 years of age, she presents hypotonia and some dysmorphic features as facial asymmetry, prominent forehead, hypertelorism, upslanting palpebral fissures, prominent nasal bridge, down-turned corners of the mouth, macroglossia, and dysmorphic ears. Congenital abnormalities include microcephaly, low-set hair, umbilical hernia, and tapering fingers. She also presents seizures, development delay (she walked at 4 years and did not speak at the age of the examination), and ID.

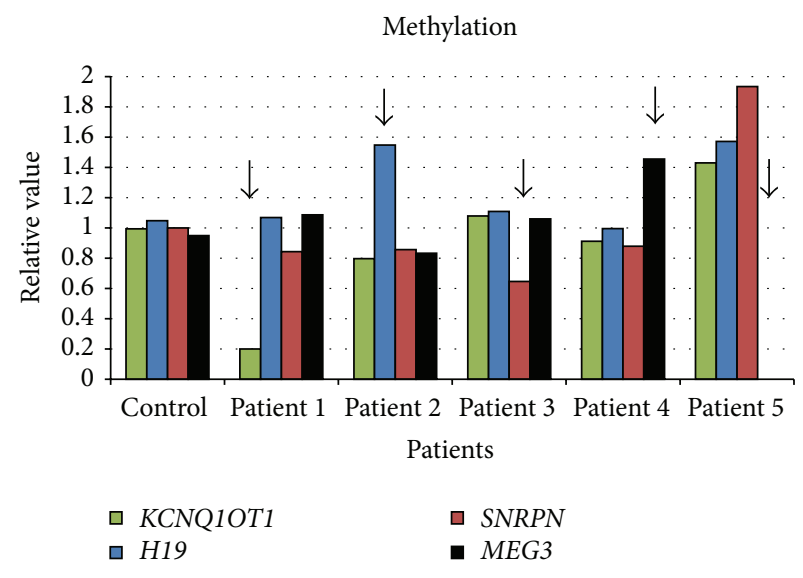

(a)

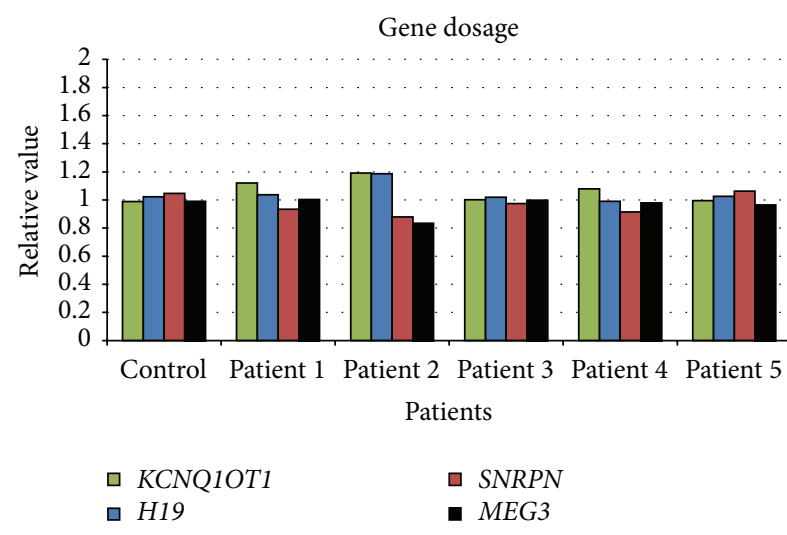

(b)

FIGURE 1: Methylation screening results. Representation of the relative value of methylation and gene dosage of the four imprinted regions (KCNQ1OT1, H19, SNRPN, and MEG3). A relative value within $1 \pm 0.2$ was considered in the normal range. The first case (left) represents a nonaltered patient. Subsequently, the results from the positive cases with different alterations of methylation are shown. A black arrow indicates the different alterations.

3.3. Patient 3. This case presents a $40 \%$ loss of methylation at SNRPN without alteration in the gene dosage (Figure 1) confirmed by MS-MLPA (SALSA ME028). Segregation analysis of chromosome 15 polymorphic markers discarded the paternal UPD. Besides, analysis by a custom array CGH, targeted to more than 400 candidate genes and a genomic backbone of $370 \mathrm{~Kb}$ resolution, did not yield any pathogenic copy number variant (GSM1527009).

The patient is the second child of nonconsanguineous healthy parents of 34 and 33 years. She was born in the 39th week of gestation with a birth weight of 3,255 g (5075 th percentile) and a length of $52 \mathrm{~cm}$ (90th percentile). At the age of seven years and 10 months she weighed $38 \mathrm{~kg}$ (>97th percentile) and her height was $135 \mathrm{~cm}$ (>97th percentile). Clinical examination noted facial dysmorphisms: hypertelorism, strabismus, dysmorphic nose, short philtrum, micrognathia, and low-set and posteriorly rotated ears. She has global developmental delay (sitting at 14 months and walking at 24 months) and ID. She only spoke single words 


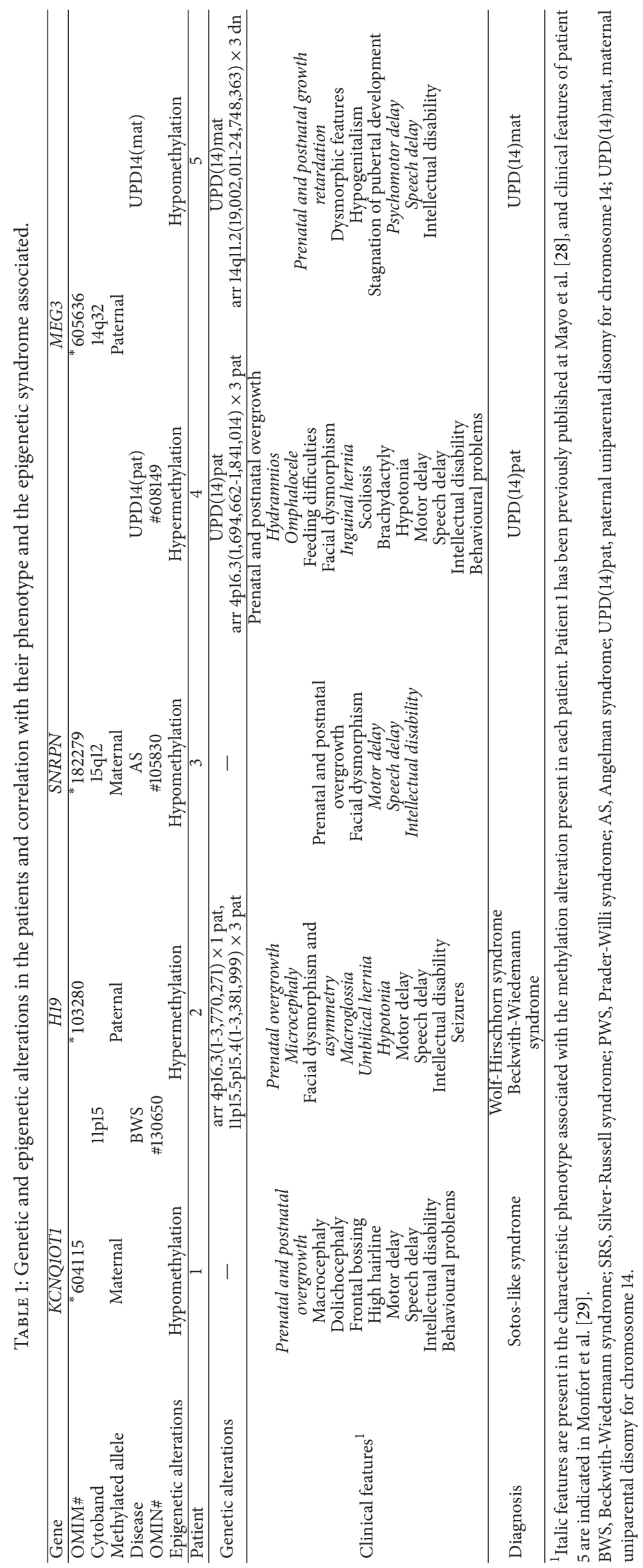


since the age of 3. Brain magnetic resonance imaging (MRI) and electroencephalography (EEG) were normal.

3.4. Patient 4. The male patient showed a hypermethylation at MEG3 without alteration in the gene dosage (Figure 1). Segregation analysis of polymorphic markers at chromosome 14 indicated a paternal uniparental disomy (UPD(14)pat) as the pathogenic mechanism of this alteration. Further studies also showed a duplication in chromosome 4 (4p16.3) of $146 \mathrm{~Kb}$ inherited from his father (arr [hg19] 4p16.3(1,694,662$1,841,014) \times 3$ pat $)($ GSM1527008) (Table 1).

$\mathrm{He}$ was the first-born child of unrelated healthy parents, a 22-year-old mother and a 27-year-old father. He was born in the 36th week by caesarean section. During the pregnancy he presents polyhydramnios, short femur, and omphalocele. His birth weight was $3,675 \mathrm{~g}$ (>90th percentile), his length was $49 \mathrm{~cm}$ (75-90th percentile), and his neonatal OFC was $36 \mathrm{~cm}$ ( $>90$ th percentile). He had neonatal hypotonia and feeding difficulties with an Apgar score of 2/5. On physical examination at 10 years, his height and weight were $132 \mathrm{~cm}$ (25th percentile) and $29 \mathrm{~kg}$ (25th percentile), respectively, and the hypotonia remained. Facial dysmorphism is seen in the form of prominent forehead, divergent strabismus, ptosis and upslanting palpebral fissures, prominent nasal bridge, thick lips, absence of some teeth, prognathism, and dysmorphic ears. Congenital abnormalities include, in addition to the omphalocele, surgically corrected at birth, tracheomalacia, patent ductus arteriosus, scoliosis, inguinal hernia, brachydactyly of the third, fourth, and fifth metacarpals, and valgus and flat feet. He presents a psychomotor development delay (walked and said his first words at 3 years and spoke simple sentences at 4) and is moderately mentally disabled. He also has nightmares and aggressiveness towards others and himself. Brain MRI and EEG results were normal.

3.5. Patient 5. This case presents a complete loss of methylation at MEG3 (14q32.2) without alteration in the gene dosage (Figure 1). By segregation analysis of polymorphic markers at chromosome 14, a maternal uniparental disomy (UPD(14)mat) was evidenced. Besides, previous assays indicated a de novo duplication at chromosome 14 of $5.7 \mathrm{Mb}$ previously published [29] (arr [hg19] 14q11.2(19,002,011$24,748,363) \times 3 \mathrm{dn})(\mathrm{GSM} 1527005)$ (Table 1). Familial segregation analysis of markers inside the duplicated area indicated the presence of two copies of the maternal alleles and one copy of the paternal allele, and FISH analysis confirmed an in situ duplication. Therefore, the patient inherited the two 14 homologues from her mother. Additionally, the duplication corresponds to the insertion of the subcentromeric region from one paternal chromosome into one maternal chromosome.

His main clinical features, previously described by Monfort et al. [29], besides psychomotor delay and mild ID, are short stature ( $<3$ rd centile) of prenatal onset, hypogenitalism, and some dysmorphic signs such as iris coloboma at the left eye, a bulbous nose, short philtrum, thin lips, clinodactyly and bilateral partial syndactyly between toes II and III, and micrognathia.

\section{Discussion}

With this study we have been able to achieve or complete the diagnosis in five patients with ID: two alterations in the methylation pattern of MEG3 as a consequence of paternal or maternal uniparental disomy for chromosome 14, one hypermethylation of $H 19$ (due to a paternal 1lp duplication), one partial loss of methylation in KCNQ1OT1, and one partial loss of methylation in SNRPN.

In addition to motor and speech delay and ID, growth anomalies (birth weight, birth length, and/or height at examination $\leq 10$ centile or $\geq 90$ centile) were present in all the five cases with different methylation anomalies detected by this screening (Table 1). By comparison, $46 \%$ of the patients in the whole series share all these symptoms. Other recurrent features in these five patients were macro- or microcephaly ( 2 cases), hypotonia ( 2 cases), hypertelorism (3 cases), and strabismus ( 2 cases).

Although patient 2 presents a genomic rearrangement responsible of the imprinting alteration, the result of the methylation test validates our strategy to detect imprinting alterations in specific loci. It is worth noting that the phenotype in the patient would be the result of two concomitant syndromes, Beckwith-Wiedemann syndrome, due to the 11 15 duplication, and the Wolf-Hirschhorn syndrome due to the 4 p16 deletion, similarly to other patients reported elsewhere [30-32]. It has been suggested that the number of cases with a CNV at the critical region of BWS could be higher than suspected and that the methylation analysis in those cases can be insufficient to provide accurate clinical diagnosis and genetic counselling.

Patients 4 and 5 showed an alteration in the methylation of $M E G 3$ due to a UPD(14) in both cases, compatible with the clinical features of the patients [33-36]. Accordingly, these results lead to a reevaluation of the $5 \mathrm{Mb}$ duplication at 14q11.2, previously considered as the cause of the clinical phenotype in patient 5 [29]. The point is that all the clinical features can now be ascribed to the maternal UPD(14), while the duplication should be considered a variant of unknown significance. Also it is worth noting that patient 4 lacks thorax deformities, which are a hallmark of upd(14)pat.

Finally the most interesting results of this study are the diagnosis of two patients with idiopathic ID. Patient 1 shows a partial loss of methylation in KCNQ1OT1 in spite of the absence of the typical features of Beckwith-Wiedemann syndrome, such as abdominal wall defects, macroglossia, hemihypertrophy, and coarse facial features; conversely he presents a Sotos-like syndrome, with the characteristic facial gestalt (downslanting palpebral fissures and pointed chin), neonatal hypotonia, large hands, or cardiac anomalies. This association was previously described by Baujat's group in a series of Sotos-like patients with no alteration in NSD1 [37]. On the other hand, patient 3 shows a partial loss of methylation in SNRPN without any other known genetic alteration. Although some clinical features of this patient might be compatible with a mild Angelman syndrome (intellectual disability and motor and speech delay), the lack of the cardinal characteristics of this disease, such as microcephaly, ataxic movements, seizures, or a distinctive behaviour [38], 
and more significantly the presence of other features not associated with Angelman syndrome, such as the overgrowth and some facial dysmorphic features (hypertelorism, short philtrum, micrognathia, and low-set ears), allow us to classify this case as another syndrome different to AS.

Both cases present a partial loss of maternal methylation. Several groups have demonstrated multilocus methylation defects in specific imprinting syndromes as in BWS $[16,17]$ or in TNDM [39]. Based on this, Azzi et al. [18, 40] proposed a multilocus loss of methylation condition where the dominant phenotype in those cases might be determined by the locus more demethylated. In our cases, the partial demethylation does not explain the phenotype observed in patients 1 and 3 so as Girardot et al. [41] suggested, other unknown imprinted loci might be affected.

Given that the pattern of differential methylation may be tissue-specific and/or time-specific, a high-throughput analysis of all the differentially methylated regions (DMRs) would not be necessarily useful in genomic DNA from blood cells. The unavailability of some tissue material as brain hampers the finding of new imprinting disorders that might be associated with ID as in those cases.

Also, one possible explanation for a global affectation at imprinting loci could be the presence of mutation at genes coding for transacting factors involved in imprinting establishment. In this sense, mutations in some genes lead to multilocus loss of methylation: ZFP57 in TNDM patients [15], NALP7 and C6orf221 in familial biparental hydatidiform mole [42, 43], or NLRP2 in a family with BWS [44]. However, a prerequisite to perform whole genome or exome sequence analysis would be the recruitment of clinically and epigenetically homogenous series of patients in order to interpret the results.

In a similar study to this work, although focused on patients with putative or confirmed imprinting disorders, $22 \%$ of patients with molecular diagnosis of an imprinting syndrome showed methylation anomalies in other loci, with no overt clinical consequences in some cases [21]. Other patients (more specifically BWS and SRS patients) associated developmental delay and other unusual congenital anomalies. In our complementary approach, focused on syndromic intellectual disability and ASD, we also found patients with methylation anomalies not associated with the corresponding syndrome but most probably reflecting new imprinting disorders with multilocus methylation anomalies. The clinical and epigenetic features that have in common the patient 1 and those reported by Baujat and colleagues [37] fully agree with this hypothesis.

\section{Conclusion}

In summary, our findings show that the complex etiology of neurodevelopmental disorders not only is limited to genetic factors, but also may be epigenetic changes that constrain or modify the phenotype of the patients. In conclusion, we found evidences of new multilocus imprinting syndromes in two patients, although further studies, not easily affordable nowadays, would be necessary to confirm this hypothesis.

\section{Conflict of Interests}

The authors declare that there is no conflict of interests regarding the publication of this paper.

\section{Acknowledgments}

This study was supported by Grants PI08/0648 and PI11/00389 (Fondo de Investigaciones Sanitarias, Ministerio de Ciencia e Innovación), FEDER (Fondo Europeo de Desarrollo Regional), and Fundación Ramón Areces. Sonia Mayo was supported by IIS La Fe/Fundación Bancaja fellowship. The authors are grateful for the collaboration of the patients, their families, and the medical specialists.

\section{References}

[1] M. Berdasco and M. Esteller, "Genetic syndromes caused by mutations in epigenetic genes," Human Genetics, vol. 132, no. 4, pp. 359-383, 2013.

[2] J. Lv, Y. Xin, W. Zhou, and Z. Qiu, "The epigenetic switches for neural development and psychiatric disorders," Journal of Genetics and Genomics, vol. 40, no. 7, pp. 339-346, 2013.

[3] J. M. Kramer and H. van Bokhoven, "Genetic and epigenetic defects in mental retardation," International Journal of Biochemistry and Cell Biology, vol. 41, no. 1, pp. 96-107, 2009.

[4] M. F. Mehler, "Epigenetic principles and mechanisms underlying nervous system functions in health and disease," Progress in Neurobiology, vol. 86, no. 4, pp. 305-341, 2008.

[5] N. C. Schanen, "Epigenetics of autism spectrum disorders," Human Molecular Genetics, vol. 15, no. 2, pp. R138-R150, 2006.

[6] B. M. Flashner, M. E. Russo, J. E. Boileau, D. W. Leong, and G. I. Gallicano, "Epigenetic factors and autism spectrum disorders," NeuroMolecular Medicine, vol. 15, no. 2, pp. 339-350, 2013.

[7] S. Rangasamy, S. R. D’Mello, and V. Narayanan, "Epigenetics, autism spectrum, and neurodevelopmental disorders," Neurotherapeutics, vol. 10, no. 4, pp. 742-756, 2013.

[8] A. A. Huzayyin, A. C. Andreazza, G. Turecki et al., "Decreased global methylation in patients with bipolar disorder who respond to lithium," International Journal of Neuropsychopharmacology, vol. 17, no. 4, pp. 561-569, 2014.

[9] E. L. Dempster, R. Pidsley, L. C. Schalkwyk et al., "Diseaseassociated epigenetic changes in monozygotic twins discordant for schizophrenia and bipolar disorder," Human Molecular Genetics, vol. 20, no. 24, Article ID ddr416, pp. 4786-4796, 2011.

[10] A. Anisowicz, H. Huang, K. I. Braunschweiger et al., "A highthroughput and sensitive method to measure Global DNA Methylation: application in Lung Cancer," BMC Cancer, vol. 8, article 222, 2008.

[11] M. F. Fraga and M. Esteller, "DNA methylation: a profile of methods and applications," BioTechniques, vol. 33, no. 3, pp. 632-649, 2002.

[12] M. S. Bartolomei, "Genomic imprinting: employing and avoiding epigenetic processes," Genes and Development, vol. 23, no. 18, pp. 2124-2133, 2009.

[13] D. Fradin, K. Cheslack-Postava, C. Ladd-Acosta et al., "Parentof-origin effects in autism identified through genome-wide linkage analysis of 16,000 SNPs," PLoS ONE, vol. 5, no. 9, Article ID e12513, pp. 1-8, 2010. 
[14] E. Kistner-Griffin, C. W. Brune, L. K. Davis, J. S. Sutcliffe, N. J. Cox, and E. H. Cook Jr., "Parent-of-origin effects of the serotonin transporter gene associated with autism," American Journal of Medical Genetics, Part B: Neuropsychiatric Genetics, vol. 156, no. 2, pp. 139-144, 2011.

[15] D. J. G. Mackay, J. L. A. Callaway, S. M. Marks et al., "Hypomethylation of multiple imprinted loci in individuals with transient neonatal diabetes is associated with mutations in ZFP57," Nature Genetics, vol. 40, no. 8, pp. 949-951, 2008.

[16] J. Bliek, G. Verde, J. Callaway et al., "Hypomethylation at multiple maternally methylated imprinted regions including PLAGL1 and GNAS loci in Beckwith-Wiedemann syndrome," European Journal of Human Genetics, vol. 17, no. 5, pp. 611-619, 2009.

[17] S. Rossignol, V. Steunou, C. Chalas et al., "The epigenetic imprinting defect of patients with Beckwitn-Wiedemann syndrome born after assisted reproductive technology is not restricted to the $11 \mathrm{p} 15$ region," Journal of Medical Genetics, vol. 43, no. 12, pp. 902-907, 2006.

[18] S. Azzi, S. Rossignol, V. Steunou et al., "Multilocus methylation analysis in a large cohort of 11p15-related foetal growth disorders (Russell Silver and Beckwith Wiedemann syndromes) reveals simultaneous loss of methylation at paternal and maternal imprinted loci," Human Molecular Genetics, vol. 18, no. 24, pp. 4724-4733, 2009.

[19] E. L. Baple, R. L. Poole, S. Mansour et al., "An atypical case of hypomethylation at multiple imprinted loci," European Journal of Human Genetics, vol. 19, no. 3, pp. 360-362, 2011.

[20] G. Perez-Nanclares, V. Romanelli, S. Mayo et al., "Detection of hypomethylation syndrome among patients with epigenetic alterations at the GNAS locus," Journal of Clinical Endocrinology and Metabolism, vol. 97, no. 6, pp. E1060-E1067, 2012.

[21] R. L. Poole, L. E. Docherty, A. Al Sayegh et al., "Targeted methylation testing of a patient cohort broadens the epigenetic and clinical description of imprinting disorders," American Journal of Medical Genetics, Part A, vol. 161, no. 9, pp. 2174-2182, 2013.

[22] J. Sambrook and D. W. Russell, Molecular Cloning: A Laboratory Manual, vol. 1, CSHL Press, New York, NY, USA, 3rd edition, 2001.

[23] S. Monfort, C. Orellana, S. Oltra, M. Roselló, M. Guitart, and F. Martínez, "Evaluation of MLPA for the detection of cryptic subtelomeric rearrangements," Journal of Laboratory and Clinical Medicine, vol. 147, no. 6, pp. 295-300, 2006.

[24] S. Mayo, S. Monfort, M. Roselló et al., "De novo interstitial triplication of MECP2 in a girl with neurodevelopmental disorder and random X chromosome inactivation," Cytogenetic and Genome Research, vol. 135, no. 2, pp. 93-101, 2011.

[25] A. López-Carrasco, S. Monfort, M. Roselló et al., "Chromosomal location of submicroscopic duplications in patients with neurodevelopmental disorders to identify cases with high risk of familial recurrence," Medicina Clinica, vol. 142, no. 12, pp. 531537, 2014.

[26] R. Edgar, M. Domrachev, and A. E. Lash, "Gene expression omnibus: NCBI gene expression and hybridization array data repository," Nucleic Acids Research, vol. 30, no. 1, pp. 207-210, 2002.

[27] F. Martínez, A. M. León, S. Monfort, S. Oltra, M. Roselló, and C. Orellana, "Robust, easy, and dose-sensitive methylation test for the diagnosis of Prader-Willi and Angelman syndromes," Genetic Testing, vol. 10, no. 3, pp. 174-177, 2006.
[28] S. Mayo, I. Garin, S. Monfort et al., "Hypomethylation of the KCNQ1OT1 imprinting center of chromosome 11 associated to Sotos-like features," Journal of Human Genetics, vol. 57, no. 2, pp. 153-156, 2012.

[29] S. Monfort, D. Blesa, M. Roselló et al., "Duplication of I4q11.2 associates with short stature and mild mental retardation: a putative relation with quantitative trait loci," American Journal of Medical Genetics, Part A, vol. 143, no. 4, pp. 382-384, 2007.

[30] C. Shuman, J. B. Beckwith, A. C. Smith, and R. Weksberg, "Beckwith-Wiedemann syndrome," in GeneReviews 1993-2014, R. A. Pagon, M. P. Adam, T. D. Bird, C. R. Dolan, C. T. Fong, and K. Stephens, Eds., University of Washington, Seattle, DC, USA, 2000.

[31] A. Battaglia, J. C. Carey, S. T. South, and T. J. Wright, "WolfHirschhorn syndrome," in GeneReviews, R. A. Pagon, M. P. Adam, T. D. Bird, C. R. Dolan, C. T. Fong, and K. Stephens, Eds., pp. 1993-2014, University of Washington, Seattle, Wash, USA, 2002.

[32] B. Baskin, S. Choufani, Y.-A. Chen et al., "High frequency of copy number variations (CNVs) in the chromosome $11 \mathrm{p} 15$ region in patients with Beckwith-Wiedemann syndrome," Human Genetics, vol. 133, no. 3, pp. 321-330, 2014.

[33] J. Mattes, B. Whitehead, T. Liehr et al., "Paternal uniparental isodisomy for chromosome 14 with mosaicism for a supernumerary marker chromosome 14," American Journal of Medical Genetics, Part A, vol. 143, no. 18, pp. 2165-2171, 2007.

[34] T. Ogata, M. Kagami, and A. C. Ferguson-Smith, "Molecular mechanisms regulating phenotypic outcome in paternal and maternal uniparental disomy for chromosome 14," Epigenetics, vol. 3, no. 4, pp. 181-187, 2008.

[35] V. R. Sutton, W. H. McAlister, T. K. Bertin et al., "Skeletal defects in paternal uniparental disomy for chromosome 14 are recapitulated in the mouse model (paternal uniparental disomy 12)," Human Genetics, vol. 113, no. 5, pp. 447-451, 2003.

[36] M. Kagami, F. Kato, K. Matsubara, T. Sato, G. Nishimura, and T. Ogata, "Relative frequency of underlying genetic causes for the development of UPD(14)pat-like phenotype," European Journal of Human Genetics, vol. 20, no. 9, pp. 928-932, 2012.

[37] G. Baujat, M. Rio, S. Rossignol et al., "Paradoxical NSD1 mutations in Beckwith-Wiedemann syndrome and 11p15 anomalies in Sotos syndrome," American Journal of Human Genetics, vol. 74, no. 4, pp. 715-720, 2004.

[38] A. I. Dagli and C. A. Williams, "Angelman syndrome," in GeneReviews, R. A. Pagon, M. P. Adam, T. D. Bird, C. R. Dolan, C. T. Fong, and K. Stephens, Eds., pp. 1993-2014, University of Washington, Seattle, Wash, USA, 1998.

[39] D. J. G. Mackay, J. M. D. Hahnemann, S. E. Boonen et al., "Epimutation of the TNDM locus and the BeckwithWiedemann syndrome centromeric locus in individuals with transient neonatal diabetes mellitus," Human Genetics, vol. 119, no. 1-2, pp. 179-184, 2006.

[40] S. Azzi, S. Rossignol, Y. Le Bouc, and I. Netchine, "Lessons from imprinted multilocus loss of methylation in human syndromes: a step toward understanding the mechanisms underlying these complex diseases," Epigenetics, vol. 5, no. 5, pp. 373-377, 2010.

[41] M. Girardot, R. Feil, and D. Llères, "Epigenetic deregulation of genomic imprinting in humans: causal mechanisms and clinical implications," Epigenomics, vol. 5, no. 6, pp. 715-728, 2013.

[42] D. A. Parry, C. V. Logan, B. E. Hayward et al., "Mutations causing familial biparental hydatidiform mole implicate C6orf221 as a possible regulator of genomic imprinting in the human 
oocyte," The American Journal of Human Genetics, vol. 89, no. 3, pp. 451-458, 2011.

[43] S. Murdoch, U. Djuric, B. Mazhar et al., "Mutations in NALP7 cause recurrent hydatidiform moles and reproductive wastage in humans," Nature Genetics, vol. 38, no. 3, pp. 300-302, 2006.

[44] E. Meyer, D. Lim, S. Pasha et al., "Germline mutation in NLRP2 (NALP2) in a familial imprinting disorder (BeckwithWiedemann syndrome)," PLoS Genetics, vol. 5, no. 3, Article ID e1000423, 2009. 

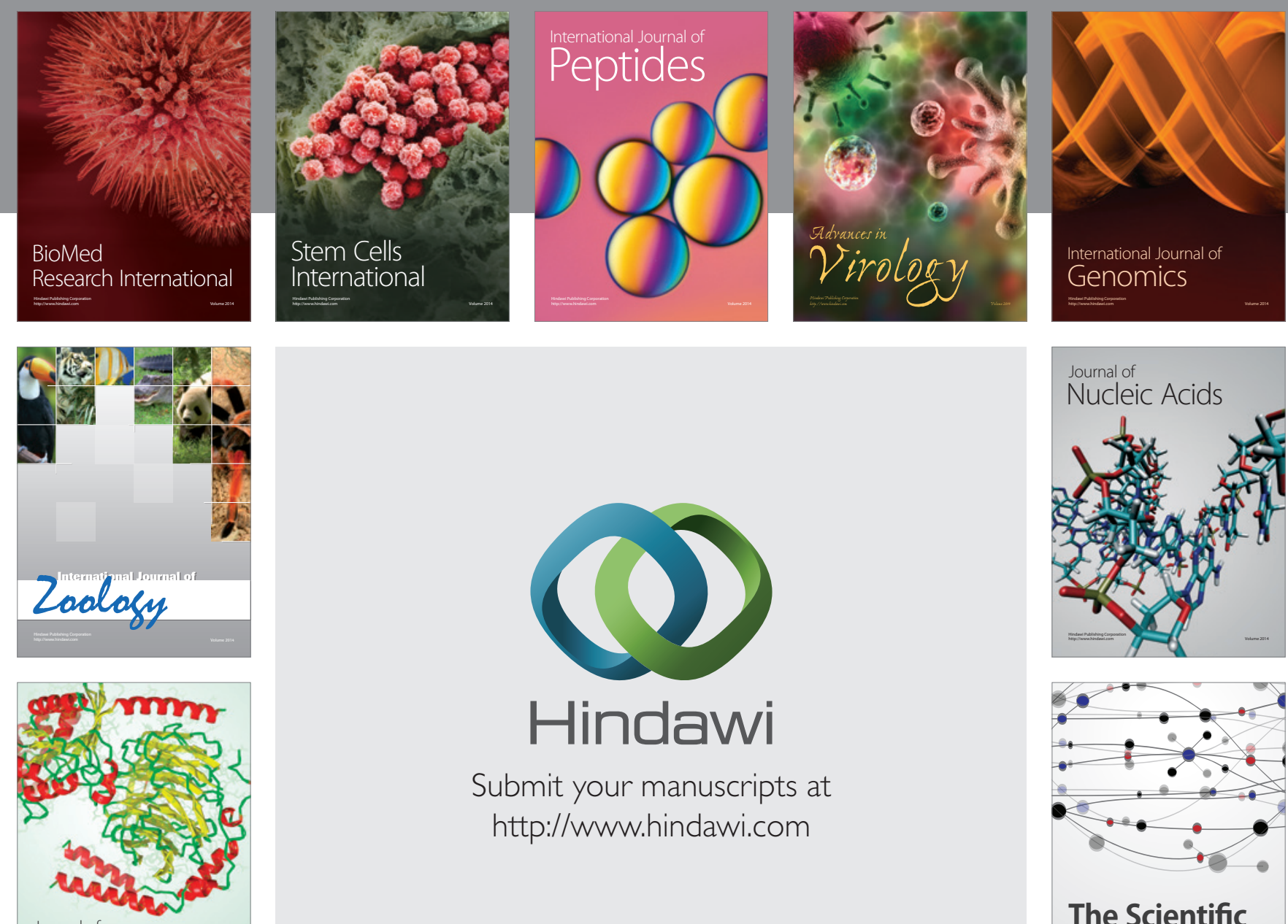

Submit your manuscripts at

http://www.hindawi.com

Journal of
Signal Transduction
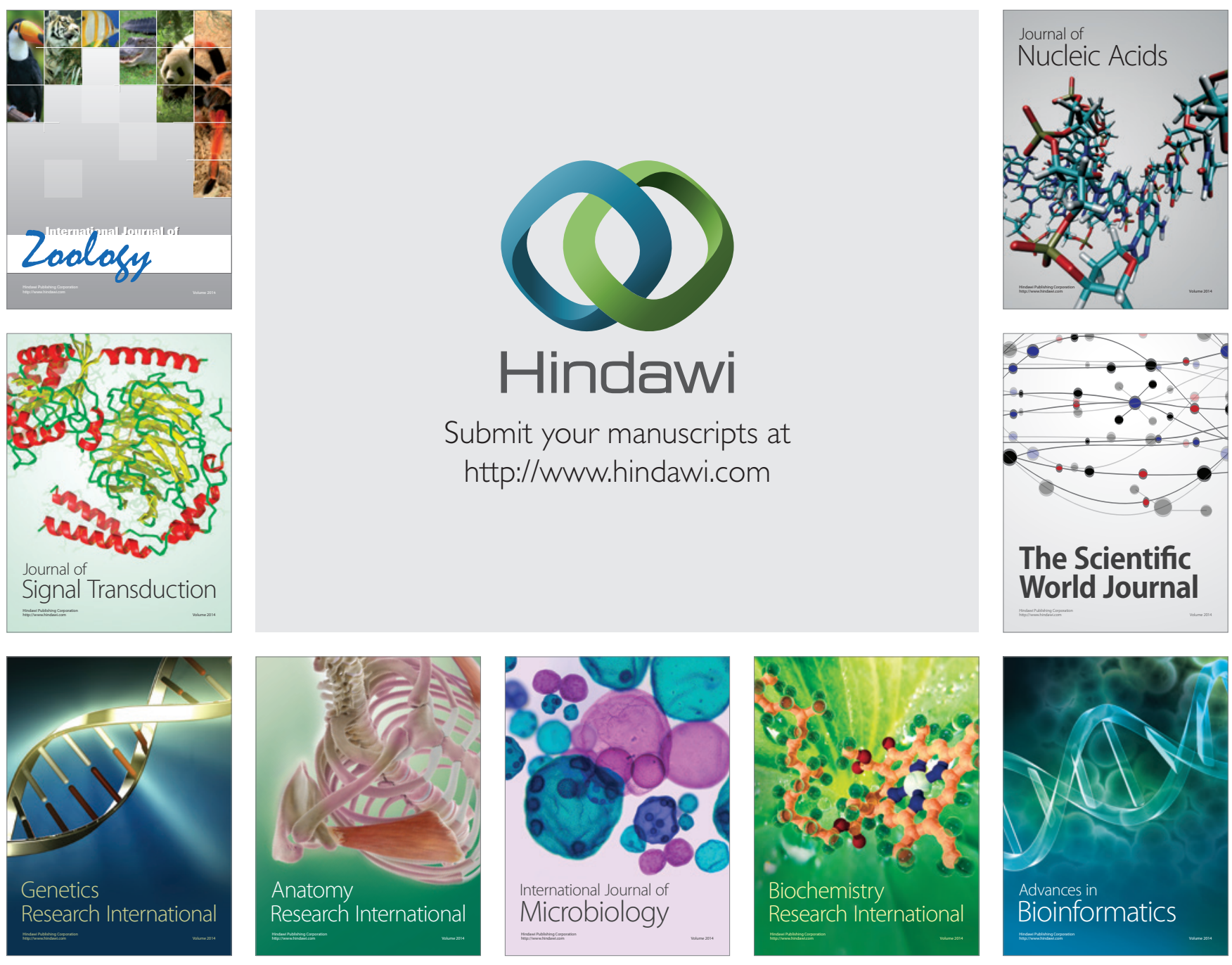

The Scientific World Journal
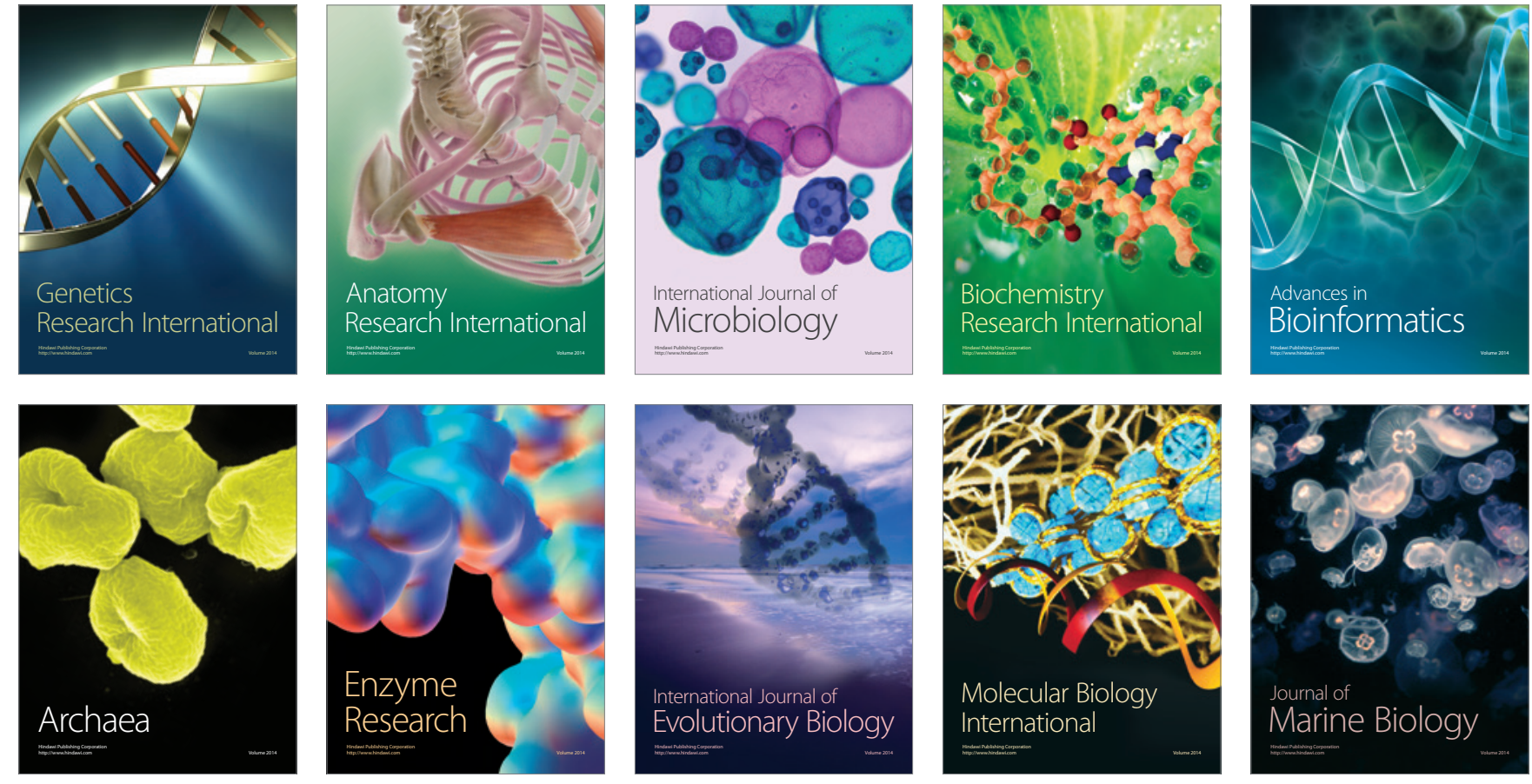